\title{
Characterizations of Modified Silica Nanoparticles(II) ; Preparation and Application of Silica Nanoparticles as a Environmentally Filler
}

\author{
Seong Kee Min ${ }^{\dagger}$, Deok Kwun Bae, Sang Bo Park, Seong Il Yoo, \\ Won Ki Lee, Chan Young Park and Soo Duk Seul* \\ Department of Polymer Engineering, Pukyong National University, Busan 608-739, Korea \\ *Department of Chemical Engineering, Dong-A University, Busan 604-714, Korea
}

(Received May 21, 2012 : Received in revised form July 26, 2012 : Accepted July 28, 2012)

\begin{abstract}
A chemical process involves polymerization within microspheres, whereas a physical process involves the dispersion of polymer in a nonsolvent. Nano-sized monodisperse microspheres are usually prepared by chemical processes such as water-based emulsions, seed suspension polymerization, nonaqueous dispersion polymerization, and precipitation polymerizations. Polymerization was performed in a four-necked, separate-type flask equipped with a stirrer, a condenser, a nitrogen inlet, and a rubber stopper for adding the initiator with a syringe. Nitrogen was bubbled through the mixture of reagents for $1 \mathrm{hr}$. before elevating the temperature. Functional silane (3-mercaptopropyl)trimethoxysilane (MPTMS) was used for the modification of silica nanoparticles and the self-assembled monolayers obtained were characterized by X-ray photoelectron spectroscopy (XPS), laser scattering system (LSS), Fourier transform infrared spectroscopy (FTIR), scanning electron microscopy (SEM), elemental analysis (EA), and thermogravimetric analysis (TGA). In addition, polymer microspheres were polymerized by radical polymerization of $\gamma$-mercaptopropyl modified silica nanoparticles (MPSN) and acrylamide monomer via precipitation polymerization; then, their characteristics were investigated. From the elemental analysis results, it can be concluded that the conversion rate of acrylamide monomer was $93 \%$ and that polyacrylamide grafted to MPSN nanospheres via the radical precipitation polymerization with AAm in ethanol solvent. The microspheres were successfully polymerized by the 'graft from' method.
\end{abstract}

Key words nanosphere, graft, silanol group, nanoparticle, degradation.

\section{Introduction}

The method to prepare microspheres varies with the use. Generally speaking, however, it can be divided into two parts; one is chemical process and the other is physical one. The chemical process is formed by polymerization within microspheres, whereas the physical process is composed by dispersion of polymer in nonsolvent. Nano-sized monodisperse microspheres are usually prepared by chemical processes such as water-based emulsions, seed suspension polymerization, nonaqueous dispersion polymerization, and precipitation polymerizations.

First and foremost, reviewing the precipitation systems which have microspheres prepared, ${ }^{1-8)}$ we find out that the preparation of microspheres is not independent of the amount of cross-linking but dependent on the initial concentration of the monomers and the properties of the solvents or solvent mixtures. For example, Goh and Stover ${ }^{1)}$

Corresponding author

E-Mail : minsk@pknu.ac.kr (S. K. Min) studied the development of polymerization products as changing the initial concentration of the monomers and the properties of solvent mixtures. As they suggested, the polymer morphology map indicated that the soluble polymer, space-filling macrogels, gels, microsphere, and coagulum might be prepared, regardless of the degree of cross-linking. These results are easy to anticipate on the basis of conventional mechanisms. Moreover, those microspheres $^{9,10)}$ were prepared only at a low initial concentration of monomers like several weight percents or less. At a higher concentration, the coagulum was obtained despite of the use of stabilizers.

Second, the preparation of microspheres rests on the polymerization temperature. At the same level of initial monomer concentration, the microspheres could not be prepared at a lower temperature, ${ }^{11)}$ even if lower temperature redox initiators were applied. For instance, microspheres of poly(N-isopropylacrylamide) (PNIPAAm) were only prepared in the temperature ranging from 52 to $78^{\circ} \mathrm{C}$ by precipitation polymerization in water. At the temperature below $50^{\circ} \mathrm{C}$, soluble polymers with lower 
molar masses were obtained.

Third, the conversion of a monomer to a soluble polymer increases the polymerization range even though most of the monomers is converted to microspheres. This represents that the polymerization in the continuous phase was independent of the microsphere formation and growth. ${ }^{12-14)}$ A variety of research groups have focused on the interaction between solvents and polymers and attempted to find the relationship between microsphere formation and properties of solvents. Yet, the interaction between solvents and monomer is not considered at this study now that it leads to the phase separation of the solvent/monomer prior to the polymerization. More specifically, it may happen although the solubility parameter of the monomer is usually used instead of the solubility of polymer. In fact, all the above-mentioned results strongly imply that the microspheres must be prepared in heterogeneous solutions in which the monomer phase is separated from the solvent, ${ }^{15-17)}$ rather than in homogeneous solutions. In this study, a functional silane (3mercaptopropyl)trimethoxysilane (MPTMS) was used for the modi-fication of silica nanoparticles and the selfassembled monolayers obtained was characterized by X-ray photoelectron spectroscopy(XPS, THERMO VG SCIENTIFIC, MultiLab 2000), laser scattering system (LSS, TOPCON Co., GLS-1000), Fourier transform infrared spectroscopy(FTIR, Perkin Elmer spectrum GX), scanning electron microscope(SEM, HITACHI, S-2400), elemental analysis(EA, Elementar, Vario macro/micro) and thermogravimetric analysis(TGA, Perkin Elmer, TGA 7, Pyris 1). In addition, polymer microspheres were polymerized by radical polymerization of MPSN and acrylamide monomer via precipitation polymerization, and then were investigate its characteristics.

\section{Experimental Procedure}

\subsection{Materials and reagents}

In this study, $\alpha, \alpha^{\prime}$-Azobis(isobutyronitrile)(AIBN) (Junsei) was used as a polymerization initiator; acrylamide monomer(Wako pure) and ethanol(SK) as solvents. MPSN was synthesized with (3-mercaptopropyl)trimethoxysilane (MPTMS).

\subsection{Method}

\subsubsection{Grafted polymerization in ethanol}

The polymerization was performed in a four-necked, separate-type flask equipped with a stirrer, a condenser, a nitrogen inlet, and a rubber stopper for adding the initiator with a syringe. Nitrogen was bubbled through the mixture of reagents for $1 \mathrm{hr}$. before elevating the temperature. We added acrylamide monomer $(7.0 \mathrm{~g})$ and ethanol $(100 \mathrm{ml})$, and then added AIBN solution $(0.03 \mathrm{~g} / 30 \mathrm{ml}$ ethanol) until $60^{\circ} \mathrm{C}$ with a syringe little by little in the prepared $\gamma$-mercaptopropyl modified silical nanoparticles(MPSN). It was polymerized at 200rpm during 14 hrs. at $60^{\circ} \mathrm{C}$. After polymerization, added $100 \mathrm{ml}$ ethanol, and then centrifuged(6000rpm for $1 \mathrm{hr}$.) to exclude unreacted monomer. This process being repeated three times, final products were obtained. The products were dried in vacuum at room temperature. As for the preparation procedure, it is summarized schematically at Scheme 1.

\subsection{Analysis and characterizations}

X-ray photoelectron spectrometer(XPS) used Thermo VG Sientific Multi LAb 2000 X-ray photoelectron spectrometer with $29.35 \mathrm{eV}$ and $\mathrm{Mg} K \alpha$ line excitation source. Using monochromator on the basis of the binding energy of $\mathrm{C} 1 \mathrm{~s}$, the analysis of binding energy was about $0.3 \mathrm{eV}$. Fourier transform infrared spectroscopy(FTIR) used Perkin Elmer spectrum GX FT-IR spectrometer. The morphology of the organo-modified silica nanoparticles was characterized by Hitachi S-2400 scanning electron microscope(SEM). The elemental analysis of $\mathrm{C}, \mathrm{N}$ and $\mathrm{H}$ was measured by Elemental vario EL instrument. The contents of reacting sites, methacrylate groups were calculated from the elemental analysis results.

\section{Results and Discussion}

\subsection{Elemental analysis}

The elemental analysis results of nanospheres of MPSN
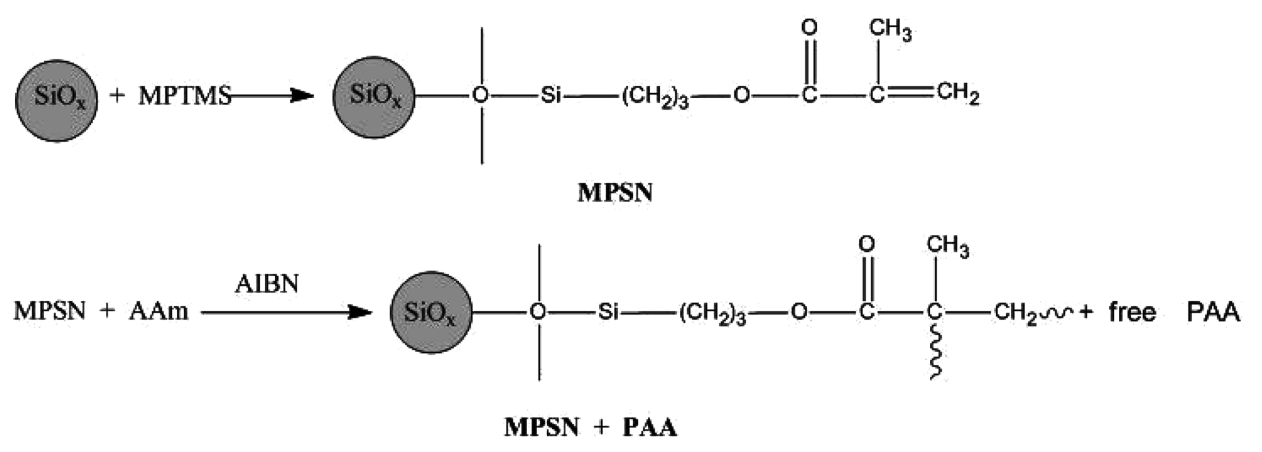

Scheme 1. The preparation procedures to MPSN+PAA. 
Table 1. Elemental analysis.

\begin{tabular}{ccc}
\hline Contents(\%) & MPSN & MPSN+PAA \\
\hline $\mathrm{C}$ & 5.29 & 21.10 \\
$\mathrm{H}$ & 1.14 & 5.262 \\
$\mathrm{~N}$ & 4.75 & 16.62 \\
\hline
\end{tabular}

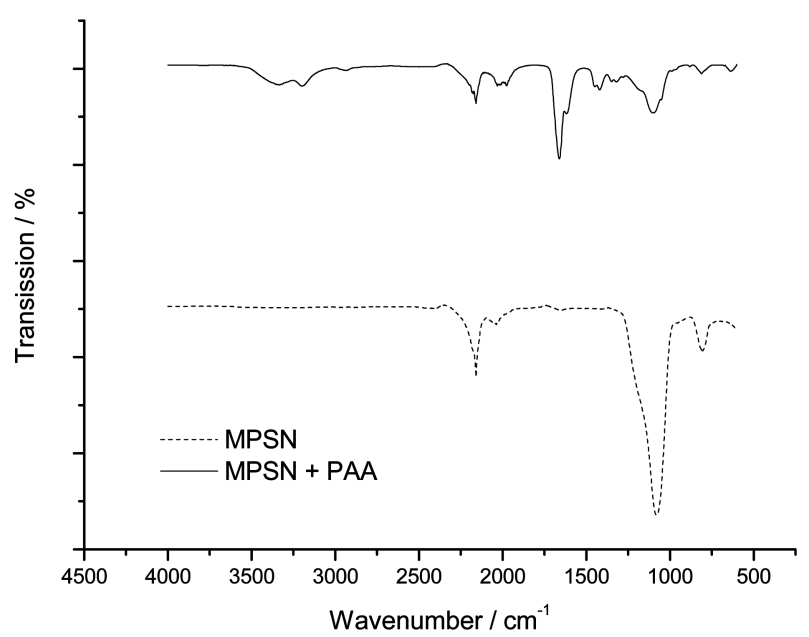

Fig. 1. Infrared spectra of the MPSN and MPSA+PAA.

and MPSN+PAA were shown in Table 1. It is found that the conversion of acrylamide monomer was $93 \%$ which is calculated from the elemental analysis results. Consequently, the findings corresponded with the mechanism information of MPSN+PAA nanosphere as can be seen in Scheme 1.

\subsection{FT-IR analysis}

Infrared spectroscopy was used as a tool to provide information on the total or partial loss of silanol groups bonded to the original silica nanoparticles and to clarify the effect caused by the immobilization of organic groups on the surfaces of organo-modified silica nanoparticles. Let us take a look at the FTIR spectrum of MPSN+PAA nanosphere(Fig. 1), N-H group, $\mathrm{C}=\mathrm{O}$ group, $\mathrm{N}-\mathrm{H}$ group and aliphatic $\mathrm{C}-\mathrm{H}$ group present at $1615 \mathrm{~cm}^{-1}, 1660 \mathrm{~cm}^{-1}$, $2935 \mathrm{~cm}^{-1}$ and $2940 \mathrm{~cm}^{-1}$, respectively. These results coincide with those of MPSN. What is more, it indicates symmetric $\mathrm{N}-\mathrm{H}$ group at $3197 \mathrm{~cm}^{-1}$. In this sense, this shows that hybrid microsphere was prepared with the proposed method.

\subsection{XPS analysis}

$\mathrm{X}$-rays are the minor wave length of electromagnetic radiant light when generate that atomic inside orbital function electron does electron transition or high energy electron reduces. The XPS spectral shifts in the binding energy of individual peaks can indicate the specific chemical species are present. The availability to use these shifts in binding energy is what makes XPS a valuable analytical
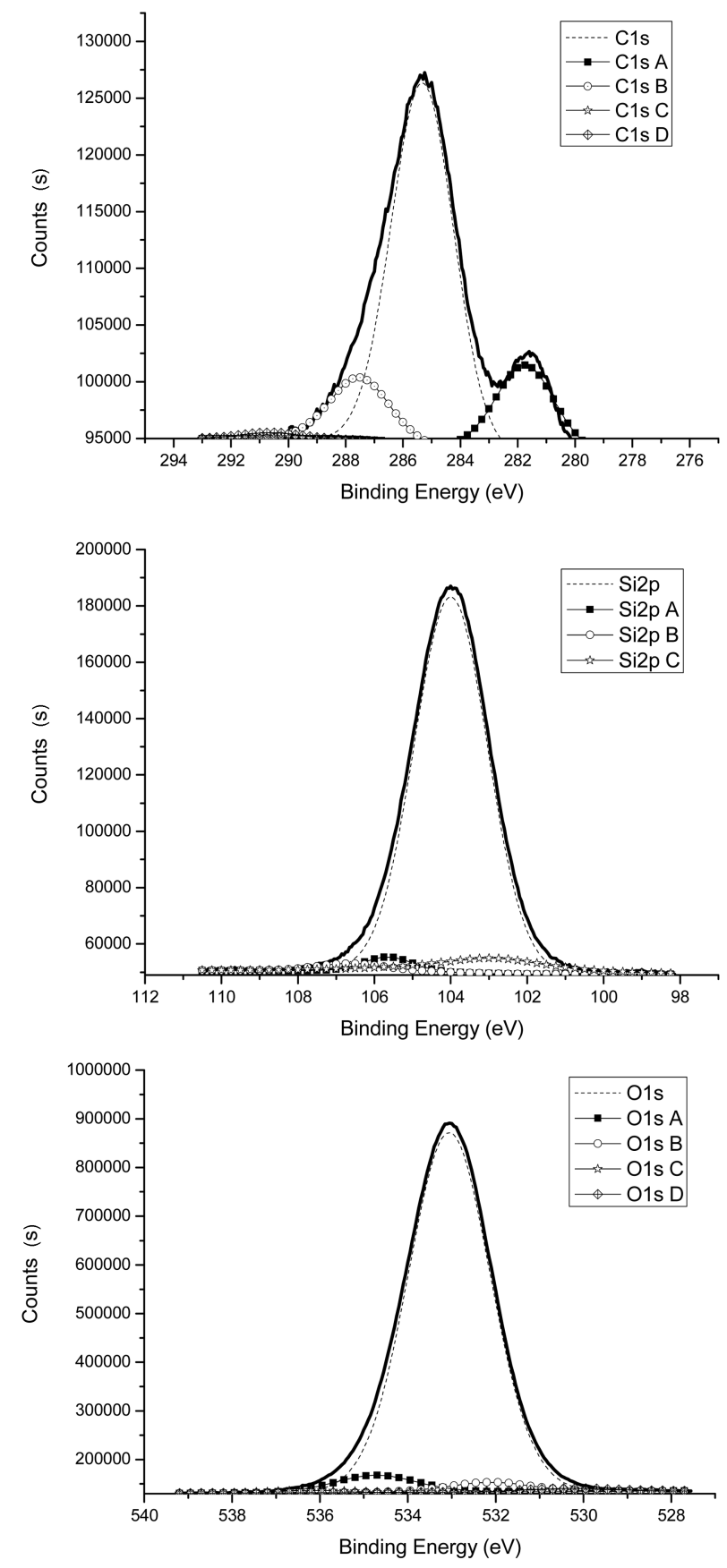

Fig. 2. XPS spectra of MPSN : (a) C1s, (b) Si2p and (c) O1s.

technique for industrial applications where bonding information is required.

\subsubsection{MPSN}

In Fig. 2, C1s, Si2p and O1s spectra of MPSN are analyzed to investigate the chemical bonding states of carbon, silicon and oxygen, and the component ratio of the quantification of functional groups. Fig. 2 shows that $\mathrm{C} 1 \mathrm{~s}$ of MPSN can resolve into four components. First $281.73 \mathrm{eV}$ is attributed to carbon such as C-H, C-C and 


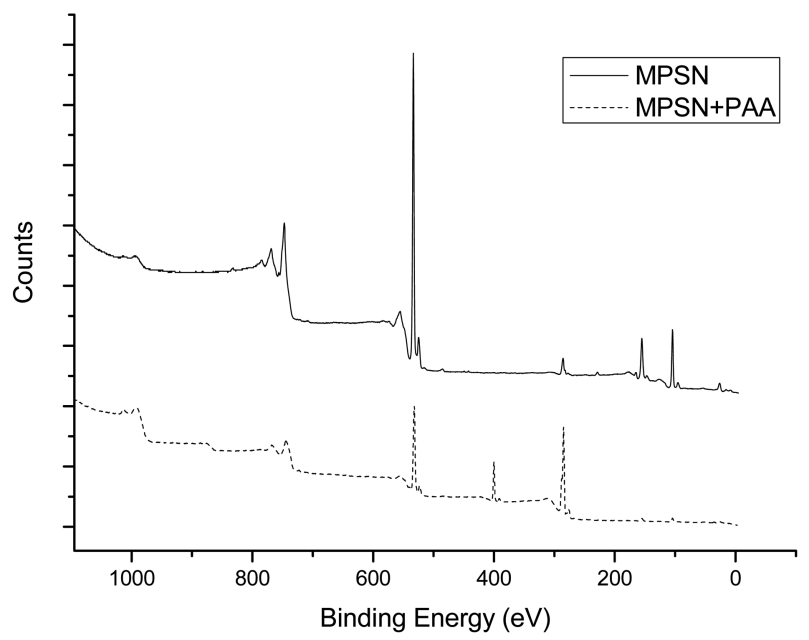

Fig. 3. XPS survey scan of the MPSN and MPSN+PAA.

$\mathrm{C}=\mathrm{C}$ in aliphatic environment. Second $290.62 \mathrm{eV}$ and third $290.34 \mathrm{eV}$ are ascribed to $(\mathrm{C}=\mathrm{O})-\mathrm{O}-\mathrm{C}$. Forth 284.52 $\mathrm{eV}$ is assigned to C-Si. As seen in Fig. 2(b), it appears that the $\mathrm{Si}-\mathrm{O}$ can be presented at $102.88 \mathrm{eV}$, and $\mathrm{Si}-\mathrm{C}$ at $105.65 \mathrm{eV}$ and $106.64 \mathrm{eV}$. Meanwhile, Fig. 2 shows that $\mathrm{O}-\mathrm{C}, \mathrm{O}=\mathrm{C}, \mathrm{O}-\mathrm{Si}$ and $\mathrm{C}-\mathrm{O}-\mathrm{Si}$ are presented at $530.28 \mathrm{eV}$, $532.12 \mathrm{eV}, 534.73 \mathrm{eV}$, and $536.23 \mathrm{eV}$ respectively. The existence of additional component characteristic of carbon element, silicon atoms bound to carbon, oxygen atoms bound to carbon represents the propyl methacrylate selfassembled monolayers which have covalent bond onto the surface of silica nanoparticles. This result accords with that of the FTIR analysis.

Next, Fig. 3 represents XPS spectra comparison of MPSN and MPSN+PAA. Spectra of MPSN and MPSN+ PAA are represented by element in Fig. 2 and Fig. 4 respectively. Peaks of MPSN are assigned to show what $\mathrm{Si}(2 \mathrm{p})$ at $110.50 \mathrm{eV}, \mathrm{O}(1 \mathrm{~s})$ at $533.50 \mathrm{eV}$ and $\mathrm{C}(1 \mathrm{~s})$ at $286.02 \mathrm{eV}$ are presented in Fig. 3. The $\mathrm{Si}(2 \mathrm{p}), \mathrm{O}(1 \mathrm{~s})$ and $\mathrm{C}(1 \mathrm{~s})$ peaks of MPSN+PAA are shown at $104.15 \mathrm{eV}$, $532.10 \mathrm{eV}$ and $285.03 \mathrm{eV}$. Particularly, the $\mathrm{N}(1 \mathrm{~s})$ peak is presented at $104.29 \mathrm{eV}$ in MPSN+PAA spectrum. This is what result of graft polymerization of nanospheres of MPSN with PAA.

\subsubsection{MPSN+PAA}

The surface component data of MPSN and MPSN+ PAA nanospheres were summarized at Fig. 4 and Table 2 with the XPS analysis. The C, N, S and O contents of
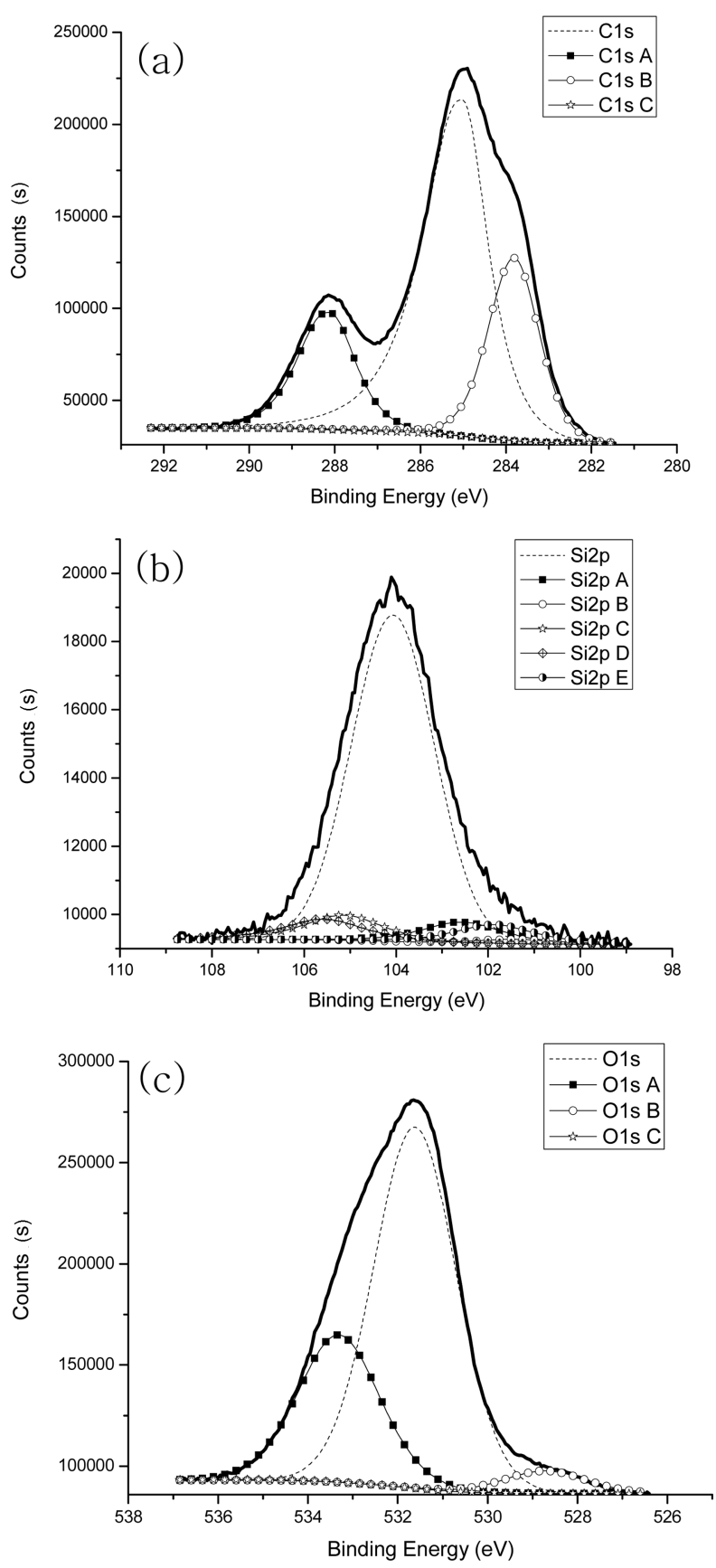

Fig. 4. XPS spectra of MPSN+PAA : (a) C1s, (b) Si2p and (c) O1s.

bare $\mathrm{SiO}_{X}$ nanoparticle were $20.77 \%, 0,30.91 \%$ and $44.70 \%$, respectively. And, the C, N, S and O contents of MPSN were $11.96 \%, 0,35.38 \%$ and $52.66 \%$, respectively. The surface element contents of $\mathrm{C}$ and $\mathrm{N}$ increase to

Table 2. The surface compositional data from XPS.

\begin{tabular}{ccccc}
\hline \multicolumn{5}{c}{ XPS analysis(at\%) } \\
\hline Samples & Si $(2 \mathrm{p})$ & $\mathrm{O}(1 \mathrm{~s})$ & $\mathrm{C}(1 \mathrm{~s})$ & $\mathrm{N}(1 \mathrm{~s})$ \\
MPSN & $35.38(110.50 \mathrm{eV})$ & $52.66(533.50 \mathrm{eV})$ & $11.96(286.02 \mathrm{eV})$ & - \\
MPSN+PAA & $1.34(104.15 \mathrm{eV})$ & $23.22(532.10 \mathrm{eV})$ & $63.44(285.03 \mathrm{eV})$ & $12.00(401.29 \mathrm{eV})$ \\
\hline
\end{tabular}


$63.44 \%$ and $12.00 \%$, while $\mathrm{Si}$ and $\mathrm{O}$ decrease to $1.34 \%$ and $23.32 \%$. The $\mathrm{SiO}, \mathrm{C}-\mathrm{O}$ and $\mathrm{O}=\mathrm{C}-\mathrm{O}$ type of $\mathrm{O} 1 \mathrm{~s}$, Si$\mathrm{C}, \mathrm{C}-\mathrm{H}, \mathrm{C}-\mathrm{C}, \mathrm{C}=\mathrm{C}, \mathrm{C}-\mathrm{O}$ and $\mathrm{O}-\mathrm{C}=\mathrm{O}$ type of $\mathrm{C} 1 \mathrm{~s}$ exist to core-level spectra of MPSN. However, $\mathrm{N}-\mathrm{C}=\mathrm{O}$ type of $\mathrm{O} 1 \mathrm{~s}$ and $\mathrm{C}-\mathrm{H}, \mathrm{C}-\mathrm{C}, \mathrm{C}=\mathrm{C}, \mathrm{C}-\mathrm{N}$ and $\mathrm{N}-\mathrm{C}=\mathrm{O}$ type of $\mathrm{C} 1 \mathrm{~s}$ only are presented to core-level spectra of MPSN+PAA microsphere. So, the variation of binding energy of O1s and $\mathrm{C} 1 \mathrm{~s}$ can only be found in microsphere of MPSN+ PAA with PAA.

\subsection{Morphological analysis}

Scanning electron microscopy is used for the morphology analysis and the images of MPSN and MPSN+ PAA. Fig. 5 shows MPSN and MPSN+PAA. MPSN has a poor dispersibility in ethanol. The diameter of MPSN+ PAA nanospheres is $20 \sim 80 \mathrm{~nm}$. This is much bigger than the $7 \mathrm{~nm}$ of bare $\mathrm{SiO}_{X}$ nanoparticles when used to prepare MPSN. It shows that the $\mathrm{SiO}_{X}$ nanoparticles are grafted and partial acrylamide monomers are polymerized by

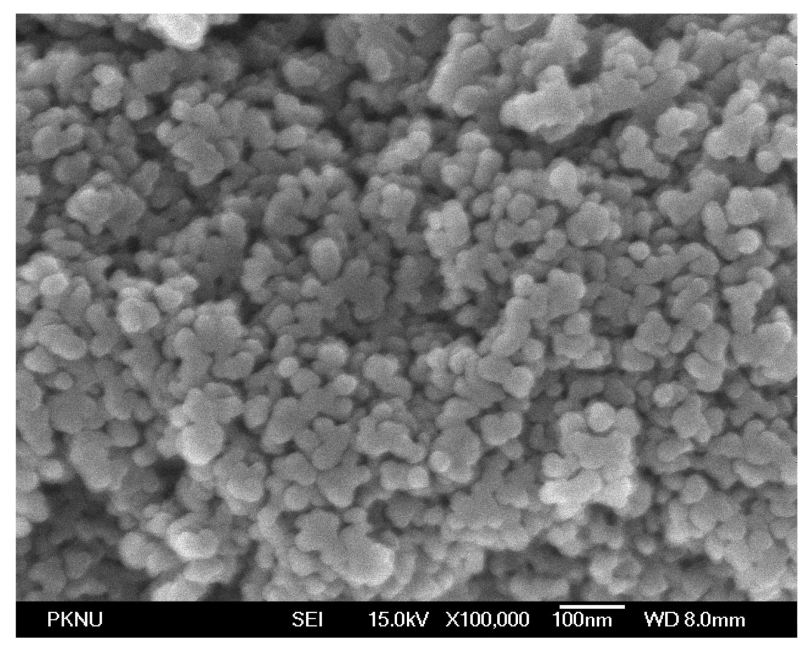

(a)

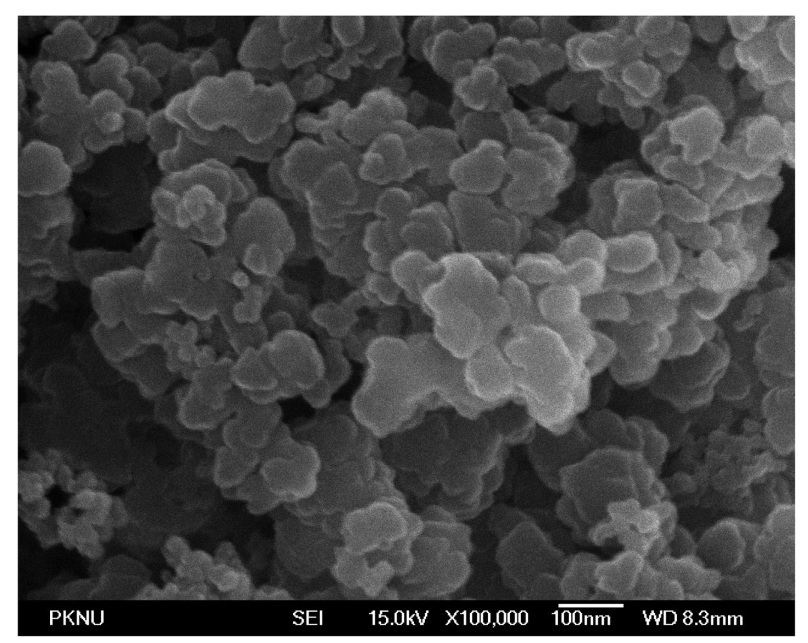

(b)

Fig. 5. SEM images of (a) MPSN and (b) MPSN+PAA.

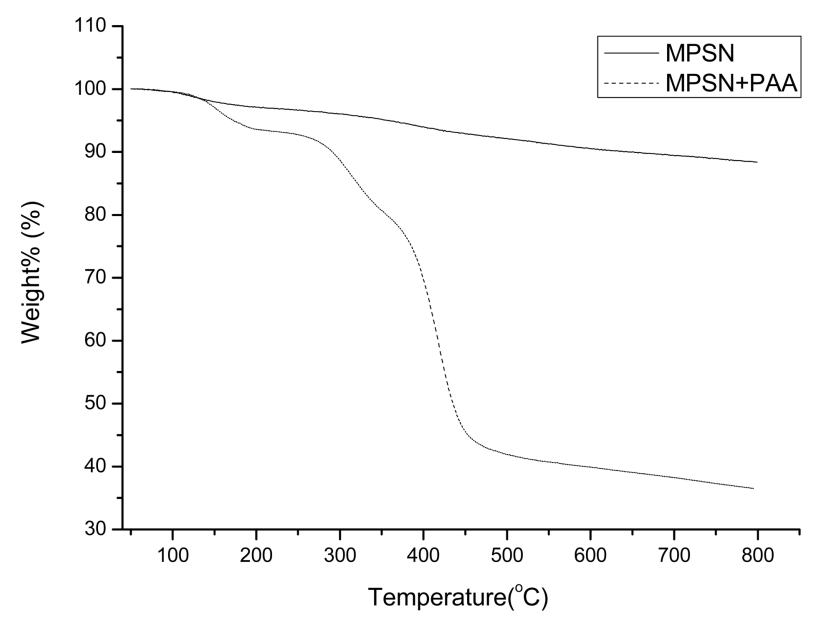

Fig. 6. TGA curve of MPSN and MPSN+PAA.

itself, and then they are absorbed to the surface of MPSN+PAA, so they are encapsulated by PAA. The result may be due to the aggregation and adsorption between MPSN+PAA and free PAA, as shown in Scheme 11. The analytical results of MPSN and MPSN+PAA particle sizes are about $42 \mathrm{~nm}$ and $70 \mathrm{~nm}$ by Electrophoretic Light Scattering Spectro photometer. These correspond with images of SEM.

\subsection{Thermal analysis}

The thermal stability of MPSN is investigated by the TGA analysis(Fig. 6). The weight loss of bare silica nanoparticles shows that the desorption of other gases have absorbed from the air at below $100^{\circ} \mathrm{C}$. There was the weight loss of MPSN at below $150^{\circ} \mathrm{C}$ but the weight of MPSN is stabilized at almost all the temperature. Nevertheless, the weight loss appears in MPSN+PAA. The reason why this happens is that grafted acrylamide and free PAA occurred the weight loss by thermal degradation.

\section{Conclusion}

Given the elemental analysis results, it can be concluded that conversion rate of acrylamide monomer was 93\%. In particular, in case of MPSN+PAA, the N(1s) was presented at $401.29 \mathrm{eV}$ by XPS analysis, and the weight loss was generated by thermal degradation. In the light of these results, the polyacrylamide grafted to MPSN nanospheres via the radical precipitation polymerization with AAm in ethanol solvent. The microspheres were successfully polymerized by 'graft from' method.

\section{References}

1. E. C. C. Goh and H. D. H. Stover, Macromolecules, 35, 
9983 (2002).

2. J. Gao and B. J. Frisken, Langmuir, 19, 5212 (2003).

3. J. Gao and B. J. Frisken, Langmuir, 19, 5217 (2003).

4. D. Gan and L. A. Lyon, Macromolecules, 35, 9634 (2002).

5. C. D. Jones and L. A. Lyon, Macromolecules, 36, 1988 (2003).

6. J. S. Downey, R. S. Frank, W. -H. Li and H. D. H. Stover, Macromolecules, 32, 2838 (1999).

7. W. -H. Li and H. D. H. Stover, J. Polymer Sci. Polymer Chem., 36, 1543 (1998).

8. Y. Naka, I. Kaetsu, Y. Yamamoto and K. Hayashi, J. Polymer Sci. Polymer Chem., 29, 1197 (1991).

9. Y. Naka and Y. Yamamoto, J. Polymer Sci. Polymer Chem., 30, 2149 (1992).

10. R. H. Pelton and P. Chibante, Colloid. Surface., 20, 247 (1986).
11. H. Kawaguchi, M. Kawahara, N. Yaguchi, F. Hoshino and Y. Ohtsuka, Polymer J., 20, 903 (1988).

12. H. Kawaguchi, Y. Yamada, S. Kataoka, Y. Morita and Y. Ohtsuka, Polymer J., 23, 955 (1991).

13. C. M. Tseng, Y. Y. Lu, M. S. El-Aasser and J. W. Vanderhoff, J. Polymer Sci. Polymer Chem., 24, 2995 (1986).

14. A. Tuncel, R. Kahraman and E. Piskin, J. Appl. Polymer Sci., 50, 303 (1993).

15. Y. Chen and H. -W. Yang, J. Polymer Sci. Polymer Chem., 30, 2765 (1992).

16. S. Kobayashi, H. Uyama, J. H. Choi and Y. Matsumoto, Polymer Int., 30, 265 (1993).

17. S. -K. Min, C. -Y. Park, W. -K. Lee, S. -D. Seul, Kor. J. Mater. Res., 22(6), 275 (2012). 